\author{
T. Umapathi \\ Ai Ching Kor \\ N. Venketasubramanian \\ C. C. Tchoyoson Lim \\ Boon Chuan Pang \\ Tseng Tsai Yeo \\ Cheng Chuan Lee \\ Poh Lian Lim \\ Kuperan Ponnudurai \\ Khoon Leong Chuah \\ Puay Hoon Tan \\ Dessmon Yeng Huoa Tai \\ Sze Peng Brenda Ang
}

\title{
Large artery ischaemic stroke in severe acute respiratory syndrome (SARS)
}

\author{
T. Umapathi, MRCP ( $凵)$. \\ N. Venketasubramanian, MMed • \\ C. C. T. Lim, FRCR - B. Ch. Pang, MRCS (Ed) \\ - T. T. Yeo, FRACS \\ Dept. of Neurology \\ National Neuroscience Institute \\ 11 Jalan Tan Tock Seng \\ Singapore 308433 \\ Fax: + 65/63577137 \\ E-Mail: \\ umapathi_thirugnanam@nni.com.sg
}

A. Ch. Kor, MRCP • C. C. Lee, MMed .

P. L. Lim, MD · K. Ponnudurai, FRCP .

D. Y. H. Tai - S. P. B. Ang, MMed

Tan Tock Seng Hospital, Singapore

11, Jalan Tan Tock Seng

Singapore 308433

K. L. Chuah, FRCPA · P. H. Tan, FRCPA

Dept. of Pathology

Singapore General Hospital

Outram Road

Singapore 169608
This paper is dedicated to the five health care workers who lost their lives in the outbreak of SARS in Singapore.

Abstract Of the 206 patients who contracted Severe Acute Respiratory Syndrome (SARS) in Singapore five developed large artery cerebral infarctions. Four patients were critically-ill and three died. Intravenous immunoglobulin was given to three patients. An increased incidence of deep venous thrombosis and pulmonary embolism was also observed among the critically-ill patients. We believe our experience warrants an increased vigilance against stroke and other thrombotic complications among critically-ill SARS patients in future outbreaks, especially if treatment such as intravenous immunoglobulin, that increases pro-thrombotic tendency, is contemplated.

Key words SARS · stroke · hypercoagulable state

\section{Introduction}

Severe Acute Respiratory Syndrome (SARS) is a highly infectious viral respiratory illness caused by a novel coronavirus [1]. It is believed to have originated from Guangdong in Southern China [2] and eventually affected about two dozen countries worldwide. The World Health Organisation (WHO) has estimated that more than eight thousand people have been affected by this illness and 774 people have died. In Singapore 32 out of the 206 patients who contracted SARS died. Almost a quarter, 48 patients, were critically-ill. Five developed large artery cerebral thrombosis. Stroke occurring in the context of viral infections is exceedingly rare. Here we report the clinical features and explore various patho- physiological mechanisms that could have contributed to cerebral thrombosis in these patients.

\section{Patients and methods}

There were 206 cases of SARS, as defined by the WHO [1], in Singapore. All except seven were managed at one institution, Tan Tock Seng Hospital (TTSH). 46 of the 199 patients were critically-ill; that is they suffered hypoxaemia, hypotension or multi-organ failure severe enough to be admitted to the intensive care unit. Two out of the seven patients managed in the other hospitals needed intensive care. Neurological complications in all SARS patients were tracked prospectively and reviewed by the first author. Table 1 summarises the salient features of the five patients with stroke. These were all patients managed at TTSH. 


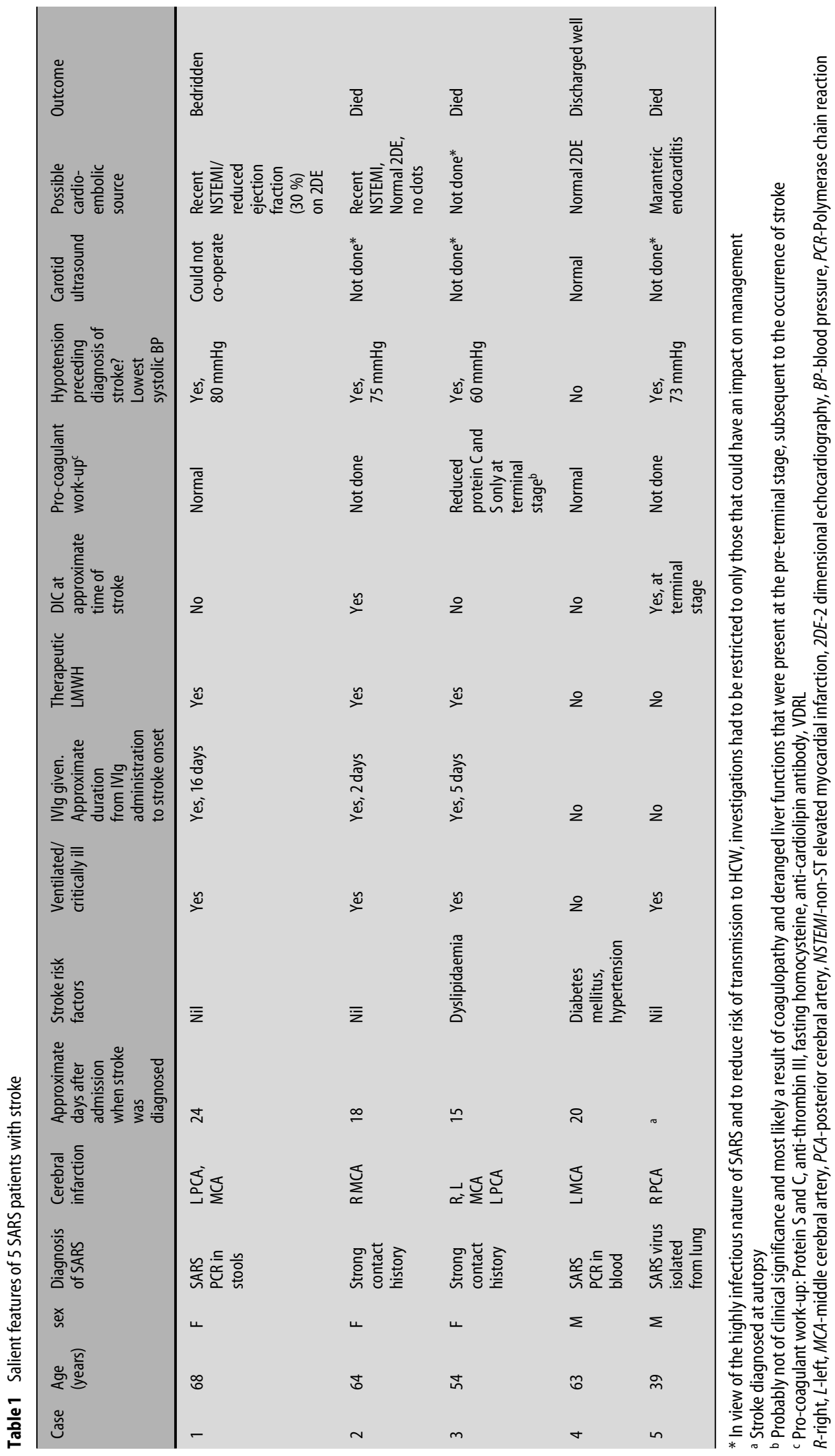




\section{Case 1}

A 68-year-old woman, with no vascular risk factors, developed SARS that was complicated by respiratory failure, non-ST elevation acute myocardial infarction (AMI), acute renal failure and nosocomial infections. As part of an evolving treatment protocol of severe SARS, she received intravenous immunoglobulin (IVIg), methylprednisolone and twice-daily subcutaneous low molecular weight heparin (LMWH). Three weeks into her hospitalization paralytic and sedative drugs were withdrawn. She did not regain consciousness. The pupillary, oculocephalic and plantar reflexes were normal. Just before this her blood pressure had decreased for a few hours to systolic $80 \mathrm{mmHg}$. Computed tomography (CT) of the brain (Fig. 1) showed infarctions in the left posterior and middle cerebral artery (PCA, MCA) territories. Aspirin was started and LMWH discontinued. She was weaned off the ventilator two months later. She remains aphasic and hemiplegic.

\section{Case 2}

A 64-year-old woman, with no stroke risk factors, contracted SARS from a health-care worker (HCW) relative. She was treated with ribavarin, IVIg and convalescent serum. She developed respiratory failure and one transient episode of hypotension (lowest recorded systolic blood pressure of $75 \mathrm{~mm} \mathrm{Hg}$ ) associated with T inversion on all electrocardiograph leads. 2D-echo showed normal ejection fraction and ventricular wall motion. No clots or vegetations were noted. She was given twice-daily LMWH that was later reduced to once a day because of a decline in haematocrit. The blood pressure improved promptly with inotropic drugs. She subsequently developed nosocomial pneumonia and disseminated intravascular coagulation (DIC). Nine days after admission to the intensive care unit (ICU), her right pupil suddenly became dilated and unreactive. The oculocephalic reflex was absent and her limbs were flaccid. Brain CT showed massive right MCA infarction with oedema and early hydrocephalus (Fig. 2). She developed central diabetes insipidus and died one week later.

\section{Case 3}

A 54-year-old female HCW, with a history of dyslipidaemia and treated hyperthyroidism, became critically-ill with SARS. She re- ceived ribavarin, IVIg and twice-daily LMWH. Fifteen days after admission she developed central diabetes insipidus. Her blood pressure dropped to systolic $60 \mathrm{mmHg}$, but responded promptly to desmopression acetate (DDAVP), intravenous fluids and inotropic drugs. A few days later her pupils suddenly dilated and showed poor reaction to light. The oculocephalic reflex was absent and her limbs were flaccid. Brain CT showed large infarctions in left PCA and bilateral MCA territories (Fig. 3). She died two days later.

\section{Case 4}

A 63-year-old man with diabetes mellitus, hypertension and ischaemic heart disease developed SARS from another patient. He did not develop respiratory failure or become critically-ill. He did not receive LWMH or IVIg. Two weeks after admission, he suddenly developed partial left hemispheric syndrome. CT brain showed a left temporo-parietal infarction.

\section{Case 5}

A 39-year-old man, with no stroke risk factors, died two weeks after contracting SARS. His illness was complicated with respiratory failure, nosocomial infections, septicaemia, DIC, AMI, and cardiac arrhythmias. He was not given LWMH or IVIg as he was one of the first few critically-ill patients with SARS in Singapore. No neurological deficits were noted before death. At autopsy, an infarction in the inferior lateral part of right occipital lobe was noted. Sterile vegetations in multiple cardiac valves, deep venous thrombosis and pulmonary embolism were detected. tients.

These findings prompted the use of LMWH in subsequent pa-

\section{Discussion}

Large artery stroke was diagnosed in five out of the 206 patients with SARS in Singapore, four of whom were critically-ill and three died. The incidence might have been higher as autopsy was not done for all deaths. A pa-

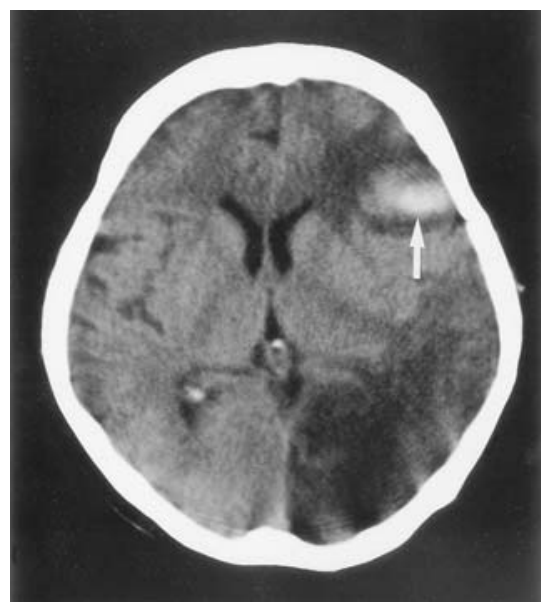

Fig. 1 Case 1. Computed tomography showed cerebral infarction involving the left posterior and middle cerebral artery territories with haemorrhage conversion (arrow)

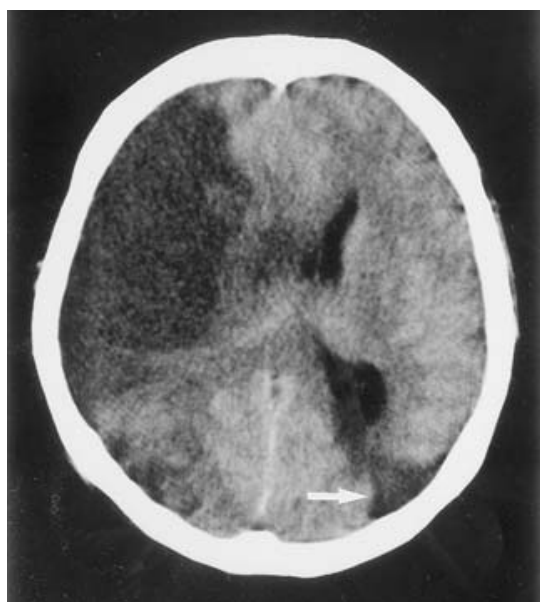

Fig. 2 Case 2. Computed tomography showed right middle cerebral artery infarction with cerebral oedema and mass effect. An older left posterior watershed infarction was also noted (arrow)

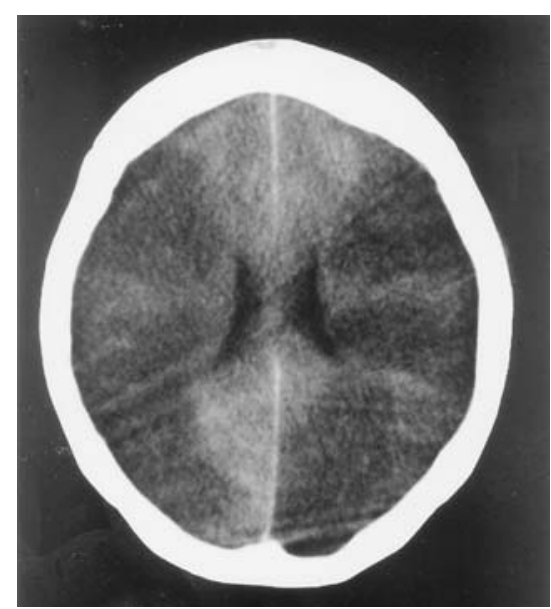

Fig. 3 Case 3. Computed tomography showed extensive cerebral infarction involving both middle cerebral and left posterior cerebral arteries 
tient with stroke and SARS was reported in a Beijing epidemiological study [3]. However, that 91-year-old patient appeared to have developed SARS after the onset of stroke. To our knowledge there have been no other reports of cerebral arterial or venous thromboses in SARS patients.

Stroke is not uncommon in criticallyill patients with multiple co-morbidity. Two of the stroke patients had cardiac dysfunction and DIC. Significant hypotension was present just before the onset of stroke in four patients (Table 1). Embolism from proximal sites, including the heart and severe hypotension in the context of sepsis could have resulted in the strokes. The apparent association between SARS and stroke therefore might be coincidental. However, about a third of criticallyill SARS patients managed at TTSH also had venous thromboembolism (including pulmonary embolism) in spite of treatment with LMWH at doses to achieve anti-Xa levels of $0.5-1.0 \mathrm{IU} / \mathrm{ml}$ (Yim CF et al., manuscript in preparation), [4]. In randomised studies of critically-ill patients, heparin has been reported to reduce the incidence of venous thromboembolism from about $30 \%$ to $15 \%[5,6]$. Four out of the eight SARS cases that underwent post-mortem examination in Singapore (this series includes case 5) had evidence of pulmonary thromboemboli [7]. A possible increase in AMI had also been observed in the Singapore cohort of SARS patients (Lim $\mathrm{IH}$, personal communication).

Furthermore, the four stroke patients who were critically-ill were not significantly older ( $56 \pm 13$ years) than all critically-ill SARS patients at TTSH (50 \pm 16 years, Anova $\mathrm{p}=0.45$ ).

The high incidence of thrombotic complications while on therapeutic doses of LMWH and the uniform pattern of large vessel ischaemic strokes (none of the patients had a lacunar stroke) that occurred in individuals with relatively few vascular risk factors suggests that a pro-coagulant state could be present in SARS. We believe that a hypercoagulable state, in tandem with factors such as systemic hypotension and cardiac dysfunction, predisposed to large cerebral arterial thromboembolism in this group of mainly critically-ill SARS patients.

The use of IVIg, known to predispose to thrombosis [8], could have contributed to the pro-thrombotic state in our patients and may explain why other groups treating SARS have not reported a similar increase in thrombosis. A retrospective four-year review of about 500 patients who received IVIg reported 16 large or medium sized artery strokes [9]. Another study found four strokes among 520 patient-days of infusion over four years [10]. However, no strokes have been reported in more than 700 patients participating in controlled trials using IVIg [8]. It is believed that IVIg-induced increase in viscosity may be inconsequential except in patients with hypercoagulable states [8]. The safety of IVIg and the role of anti-Xa monitored doses of LMWH in criti- cally-ill SARS patients should therefore be studied in future outbreaks.

Other viral infections, such as varicella zoster [11], cytomegalovirus [12], parvovirus [13] and human immunodeficiency virus have been associated with stroke. Recent bacterial and viral infections have also been reported to be independent risk factors for stroke [14]. Overall, the incidence of stroke complicating infections is exceedingly rare. Even in a common illness such as chicken pox, cerebral thrombosis, typically of large vessels, is estimated to occur in only one out of 6500 infections [11]. The co-morbidity in our patients might have contributed to the relatively high incidence of about one stroke in 42 SARS infections.

Various hypotheses have been suggested to explain the apparent link between viruses and cerebrovascular disease.Virus-induced inflammation of the vessel wall is believed to be responsible for stroke associated with chicken pox and herpes zoster [15]. Stroke and arteritis have also been documented in animal viral infections. This may be pertinent as the SARS coronavirus is widely believed to have evolved from a yet unidentified animal virus [16]. Examples of stroke in animal viral infections include malignant catarrhal fever (herpes virus), Aleutian disease (parvovirus) and border disease (pestivirus) [17]. The equine arteritis virus, an arterivirus belonging to the same order as coronaviruses, causes lymphocytic infiltration, necrosis of smooth muscle and occlusion of vessel wall. Electronmicrographs show virions within endothelial cells [18]. However, we have not been able to demonstrate SARS virions within endothelial cells or causing primary endothelial injury (data not shown). We also did not perform transcranial Doppler examinations to identify vaso-spasm, because the highly infectious nature of SARS and the increased incidence of nosocomial transmission among health care workers restricted investigations to only those that could have a direct impact on the patients' management.

Alternatively, the SARS coronavirus may have an effect similar to herpes simplex, which has been shown to reduce heparan sulfate, anti-thrombin III binding, prostacyclin, thrombomodulin [19]; and enhance thrombin formation, platelet binding [20] and tissue factor expression [19]. In this regard, the discovery of the prothrombinase gene in the SARS viral genome requires further study (Lai MMC, personal communication).

In summary, we report five SARS patients with stroke. In these patients with multiple co-morbidity we cannot be certain of the contribution of SARS coronavirus to the development of stroke. However, we believe our experience should alert others managing criticallyill SARS patients in future outbreaks to be vigilant against an increase in thrombotic complications including stroke, especially if intravenous immunoglobulin is being used for treatment. 


\section{References}

1. NN (2003) Severe acute respiratory syndrome (SARS). Wkly Epidemiol Rec 78:81-83

2. Zhong NS, Zheng BJ, Li YM, Poon Xie ZH, Chan KH, Li PH (2003) Epidemiology and cause of severe acute respiratory syndrome (SARS) in Guangdong, People's Republic of China, in February 2003. Lancet 362:1353-1358

3. Xie SY, Zeng G, Lei J, Li Q, Li HB, Jia QB (2003) Analyses on one case of severe acute respiratory syndrome 'super transmitter' and chain of transmission. Zhonghua Liu Xing Xue Za Zhi 24:449-453

4. Lew TW, Kwek TK, Tai D, Earnest A, Loo S, Singh K, Kwan KM, Chan Y, Yim CF, Bek SL, Kor AC, Yap WS, Chelliah YR, Lai YC, Goh SK (2003) Acute respiratory distress syndrome in critically ill patients with severe acute respiratory syndrome. JAMA 290:374-380

5. Fraisse F, Holzapfel L, Couland JM, Simonneau G, Bedock B, Feissel M et al. (2000) Nadroparin in the prevention of deep vein thrombosis in acute decompensated COPD. The Association of Non-University Affiliated Intensive Care Specialist Physicians of France. Am J Respir Crit Care Med 161(4 Pt 1):1109-1114

6. Kapoor M, Kupfer YY, Tessler S (1999) Subcutaneous heparin prophylaxis significantly reduces the incidence of venous thromboembolic events in the critically ill. Crit Care Med 27:A69
7. Chong PY, Chui P, Ling AE, Franks TJ, Tai DY, Leo YS, Kaw GJ, Wansaicheong G, Chan KP, Ean Oon LL, Teo ES, Tan KB, Nakajima N, Sata T, Travis WD (2004) Analysis of deaths during the severe acute respiratory syndrome (SARS) epidemic in Singapore: challenges in determining a SARS diagnosis. Arch Pathol Lab Med 128:195-204

8. Dalakas MC, Clark WM (2003) Strokes, thromboembolic events, and IVIg: Rare incidents blemish an excellent safety record. Neurology 60(11): 1736-1737

9. Caress JB, Cartwright MS, Donofrio PD, Peacock JE Jr (2003) The clinical features of 16 cases of stroke associated with administration of IVIg. Neurology 60:1822-1824

10. Okuda D, Flaster M, Frey J, Sivakumar K (2003) Arterial thrombosis induced by IVIg and its treatment with tPA. Neurology 60:1825-1826

11. Ichiyama T, Houdou S, Kisa T, Ohno K, Takeshita K (1990) Varicella with delayed hemiplegia. Pediatr Neurol 6:279-281

12. Koeppen AH, Lansing LS, Peng SK, Smith RS (1981) Central nervous system vasculitis in cytomegalovirus infection. J Neurol Sci 51:395-410

13. Guidi B, Bergonzini P, Crisi G, Frigieri G, Portolani M (2003) Case of stroke in a 7-year-old male after parvovirus B19 infection. Pediatr Neurol 28:69-71
14. Grau AJ, Buggle F, Becher H, Zimmermann E, Spiel M, Fent T, Maiwald M, Werle E, Zorn M, Hengel H, Hacke W (1998) Recent bacterial and viral infection is a risk factor for cerebrovascular ischemia: clinical and biochemical studies. Neurology 50:196-203

15. Doyle PW, Gibson G, Dolman CL (1983) Herpes zoster ophthalmicus with contralateral hemiplegia: identification of cause. Ann Neurol 14:84-85

16. Centers for Disease Control and Prevention (2003) Prevalence of IgG antibody to SARS-associated coronavirus in animal traders - Guangdong Province. MMWR Morb Mortal Wkly Rep 52:986-987

17. Johnson RT (1998) Cerebrovascular disease in animals. In: Johnson RT (ed) Viral infections of the nervous system. Lippincott-Raven, Philadelphia, pp 407-409

18. Jones TC, Doll ER, Bryans JT (1957) The lesions of equine viral arteritis. Cornell Vet 47:52-68

19. Key NS, Vercellotti GM, Winkelmann JC, Moldow CF, Goodman JL, Esmon NL et al. (1990) Infection of vascular endothelial cells with herpes simplex virus enhances tissue factor activity and reduces thrombomodulin expression. Proc Natl Acad Sci U S A 87:7095-7099

20. Visser MR, Tracy PB, Vercellotti GM, Goodman JL, White JG, Jacob HS (1988) Enhanced thrombin generation and platelet binding on herpes simplex virus-infected endothelium. Proc Natl Acad Sci U S A 85:8227-8230 